\title{
Operator Characterizations and Some Properties of $g$-Frames on Hilbert Spaces
}

\author{
Xunxiang Guo \\ Department of Mathematics, Southwestern University of Finance and Economics, Chengdu 611130, China \\ Correspondence should be addressed to Xunxiang Guo; guoxunxiang@yahoo.com
}

Received 30 May 2013; Accepted 22 September 2013

Academic Editor: Simone Secchi

Copyright (C) 2013 Xunxiang Guo. This is an open access article distributed under the Creative Commons Attribution License, which permits unrestricted use, distribution, and reproduction in any medium, provided the original work is properly cited.

Given the $g$-orthonormal basis for Hilbert space $H$, we characterize the $g$-frames, normalized tight $g$-frames, and $g$-Riesz bases in terms of the $g$-preframe operators. Then we consider the transformations of $g$-frames, normalized tight $g$-frames, and $g$-Riesz bases, which are induced by operators and characterize them in terms of the operators. Finally, we discuss the sums and $g$-dual frames of $g$-frames by applying the results of characterizations.

\section{Introduction}

A sequence $\left(f_{i}\right)_{i \in I}$ of elements of a Hilbert space $H$ is called a frame for $H$ if there are constants $A, B>0$ so that

$$
A\|f\|^{2} \leq \sum_{i \in I}\left|\left\langle f, f_{i}\right\rangle\right|^{2} \leq B\|f\|^{2}, \quad \forall f \in H
$$

The numbers $A ; B$ are called the lower (resp., upper) frame bounds. The frame is a tight frame if $A=B$ and a normalized tight frame if $A=B=1$.

The concept of frame first appeared in the late 40 s and early 50s (see, [1-3]). The development and study of wavelet theory during the last decades also brought new ideas and attentions to frames because of their close connections. There are many related references on this topic, see; [4-8].

In [9], Sun raised the concept of $g$-frame as follows, which generalized the concept of frame extensively. A sequence $\left\{\Lambda_{i} \in B\left(H, H_{i}\right): i \in N\right\}$ is called a $g$-frame for $H$ with respect to $\left\{H_{i}: i \in N\right\}$, which is a sequence of closed subspaces of a Hilbert space $K$, if there exist two positive constants $A$ and $B$ such that for any $f \in H$

$$
A\|f\|^{2} \leq \sum_{i \in N}\left\|\Lambda_{i} f\right\|^{2} \leq B\|f\|^{2} .
$$

We simply call $\left\{\Lambda_{i}: i \in N\right\}$ a $g$-frame for $H$ whenever the space sequence $\left\{H_{i}: i \in N\right\}$ is clear. The tight $g$-frame and normalized tight $g$-frame are defined similarly. We call $\left\{\Lambda_{i}: i \in N\right\}$ a $g$-frame sequence, if it is a $g$-frame for $\overline{\operatorname{span}}\left\{\Lambda_{i}^{*}\left(H_{i}\right)\right\}_{i \in N}$. We call $\left\{\Lambda_{i}: i \in N\right\}$ a $g$-Bessel sequence, if only the right inequality is satisfied. Recently, $g$-frames in Hilbert spaces have been studied intensively; for more details see $[10-14]$ and the references therein.

Constructing frames and g-frames is an interesting problem in frame theory and it is also useful in applications. In this respect, many mathematicians considered the algebraic operations among frames, which allows us to construct a large number of new frames from existing frames. For more details, see [15-18] and the references therein. In this paper, we will consider the algebraic operations among $g$-frames; in particular, we will consider the direct sums of $g$-frames and general sums of $g$-frames. We also will consider the transforms of $g$-frames and their dual $g$-frames.

\section{2. $g$-Preframe Operators and Transformations of $g$-Frames}

In this section, we introduce the $g$-preframe operators and use them to characterize $g$-frames. We also discuss the transformations of $g$-frames. Since any countable infinite sequence can be made as one indexed by natural number $N$, so all infinite sequences in this paper are assumed to be 
indexed by $N$. For each sequence $\left\{H_{i}: i \in N\right\}$, we define the space $\left(\sum_{i \in N} \oplus H_{i}\right)_{l_{2}}$ by

$$
\begin{aligned}
&\left(\sum_{i \in N} \oplus H_{i}\right)_{l_{2}} \\
& \quad=\left\{\left\{f_{i}\right\}_{i \in N}: f_{i} \in H_{i}, i \in N, \sum_{i \in N}\left\|f_{i}\right\|^{2}<+\infty\right\},
\end{aligned}
$$

with the inner product defined by

$$
\left\langle\left\{f_{i}\right\},\left\{g_{i}\right\}\right\rangle=\sum_{i \in N}\left\langle f_{i}, g_{i}\right\rangle .
$$

Definition 1 (see, [9]). Let $\Lambda_{i} \in B\left(H, H_{i}\right), i \in N$.

(i) If $\left\{f: \Lambda_{i} f=0 ; i \in N\right\}=\{0\}$, then we say that $\left\{\Lambda_{i}\right.$ : $i \in N\}$ is $g$-complete.

(ii) If $\left\{\Lambda_{i}: i \in N\right\}$ is $g$-complete and there are positive constants $A$ and $B$ such that for any finite subset $J \subset N$ and $g_{j} \in H_{j}, j \in J$,

$$
A \sum_{j \in J}\left\|g_{j}\right\|^{2} \leq\left\|\sum_{j \in J} \Lambda_{j}^{*} g_{j}\right\|^{2} \leq B \sum_{j \in J}\left\|g_{j}\right\|^{2},
$$

then we say that $\left\{\Lambda_{i}: i \in N\right\}$ is a $g$-Riesz basis for $H$ with respect to $\left\{H_{i}: i \in N\right\}$.

(iii) We say $\left\{\Lambda_{i}: i \in N\right\}$ is a $g$-orthonormal basis for $H$ with respect to $\left\{H_{i}: i \in N\right\}$ if it satisfies the following:

$$
\begin{gathered}
\left\langle\Lambda_{i}^{*} g_{i}, \Lambda_{j}^{*} g_{j}\right\rangle=\delta_{i j}\left\langle g_{i}, g_{j}\right\rangle, \quad \forall i, j \in N, g_{i} \in H_{i}, g_{j} \in H_{j}, \\
\sum_{j \in N}\left\|\Lambda_{j} f\right\|^{2}=\|f\|^{2}, \quad \forall f \in H .
\end{gathered}
$$

Remark 2. It is obvious that any $g$-frame $\left\{\Lambda_{i}: i \in N\right\}$ is $g$ complete and any $g$-orthonormal basis is a normalized tight $g$-frame.

Lemma 3 (see [11]). $\left\{\Lambda_{i} \in B\left(H, H_{i}\right), i \in N\right\}$ is g-complete if and only if $\overline{\operatorname{span}}\left\{\Lambda_{i}^{*}\left(H_{i}\right)\right\}_{i \in N}=H$.

Lemma 4 (see, [11]). $\left\{\Lambda_{i} \in B\left(H, H_{i}\right), i \in N\right\}$ is a g-Bessel sequence for $H$ with bound $B$, if and only if the operator

$$
T:\left(\sum_{i \in N} \oplus H_{i}\right)_{l_{2}} \longrightarrow H
$$

defined by $T\left(\left\{g_{i}\right\}\right)=\sum_{j \in N} \Lambda_{j}^{*}\left(g_{j}\right)$ is a well-defined bounded operator with $\|T\| \leq \sqrt{B}$.

Lemma 5. Let $\left\{\theta_{i} \in B\left(H, H_{i}\right): i \in N\right\}$ be a g-orthonormal basis for $H$ with respect to $\left\{H_{i}: i \in N\right\}$; then the sequence $\left\{\Lambda_{i} \in B\left(H, H_{i}\right): i \in N\right\}$ is a $g$-Bessel sequence with respect to $\left\{H_{i}: i \in N\right\}$ if and only if there is a unique bounded operator $V: H \rightarrow H$ such that $\Lambda_{i}=\theta_{i} V^{*}$, for all $i \in N$.
Proof. $\Rightarrow$ : Since $\left\{\theta_{i} \in B\left(H, H_{i}\right): i \in N\right\}$ is a $g$-orthonormal basis for $H,\left\{\theta_{i} f: i \in N\right\} \in\left(\sum_{i \in N} \oplus H_{i}\right)_{l_{l}}$, for any $f \in H$. If $\left\{\Lambda_{i} \in B\left(H, H_{i}\right): i \in N\right\}$ is a $g$-Bessel sequence, then the operator

$$
V: H \longrightarrow H, \quad V f=\sum_{i \in N} \Lambda_{i}^{*} \theta_{i} f
$$

is well-defined and bounded operator by Lemma 5 . Also by the definition of $g$-orthonormal basis, it is easy to see that $\theta_{i} \theta_{j}^{*} g=\delta_{i j} g$. So

$$
V \theta_{j}^{*} g=\sum_{i \in N} \Lambda_{i}^{*} \theta_{i} \theta_{j}^{*} g=\Lambda_{j}^{*} \theta_{j} \theta_{j}^{*} g=\Lambda_{j}^{*} g
$$

for all $g \in H_{j}, j \in N$. Hence $V \theta_{j}^{*}=\Lambda_{j}^{*}$, which implies that $\theta_{j} V^{*}=\Lambda_{j}, j \in N$. Suppose $V_{1}, V_{2} \in B(H)$ and $\theta_{i} V_{1}^{*}=\theta_{i} V_{2}^{*}=$ $\Lambda_{i}$ for any $i \in N$. Then for any $f \in H, g_{i} \in H_{i}$, we have $\left\langle\theta_{i} V_{1}^{*} f, g_{i}\right\rangle=\left\langle\theta_{i} V_{2}^{*} f, g_{i}\right\rangle$, that is, $\left\langle V_{1}^{*} f, \theta_{i}^{*} g_{i}\right\rangle=\left\langle V_{2}^{*} f, \theta_{i}^{*} g_{i}\right\rangle$ Since $\operatorname{span}\left\{\theta_{i}^{*}\left(H_{i}\right)\right\}_{i \in N}=H$ by Lemma $3, V_{1}^{*} f=V_{2}^{*} f$, which means that $V_{1}=V_{2}$. Thus the operator $V$ is unique.

$\Leftarrow$ : Since $\Lambda_{i}=\theta_{i} V^{*}$, for all $i \in N$, for any $f \in H$, we have

$$
\sum_{i \in N}\left\|\Lambda_{i} f\right\|^{2}=\sum_{i \in N}\left\|\theta_{i} V^{*} f\right\|^{2}=\left\|V^{*} f\right\|^{2} \leq\left\|V^{*}\right\|^{2}\|f\|^{2} .
$$

So $\left\{\Lambda_{i} \in B\left(H, H_{i}\right): i \in N\right\}$ is a $g$-Bessel sequence with respect to $\left\{H_{i}: i \in N\right\}$.

Definition 6. Let $\left\{\theta_{n}: n \in N\right\}$ be a $g$-orthonormal basis for $H,\left\{\Lambda_{i} \in B\left(H, H_{i}\right): i \in N\right\}$ is a $g$-Bessel sequence for $H$ with respect to $\left\{H_{i}: i \in N\right\}$, and the g-preframe operator associated with $\left\{\Lambda_{i}: i \in N\right\}$ is the operator $V \in B(H)$ such that $\theta_{n} V^{*}=\Lambda_{n}$ for any $n \in N$. The g-frame operator $S$ associated with $\left\{\Lambda_{i} \in B\left(H, H_{i}\right): i \in N\right\}$ is defined by $S f=\sum_{i \in N} \Lambda_{i}^{*} \Lambda_{i} f$.

Remark 7. It is well known that if $\left\{\Lambda_{i} \in B\left(H, H_{i}\right): i \in N\right\}$ is a $g$-frame, then $S$ is a well-defined positive invertible operator on $H$ and $\left\{\widetilde{\Lambda}_{i}=\Lambda_{i} S^{-1}: i \in N\right\}$ is the canonical dual $g$ frame of $\left\{\Lambda_{i}: i \in N\right\}$; for details see [9]. In this case, for any $f \in H$, we have the Fourier series type decomposition: $f=$ $\sum_{i \in N} \Lambda_{i}^{*} \widetilde{\Lambda}_{i} f=\sum_{i \in N} \widetilde{\Lambda}_{i}^{*} \Lambda_{i} f$. In general, there are many $g$ frames $\left\{\Gamma_{i}: i \in N\right\}$ for which the formula $f=\sum_{i \in N} \Lambda_{i}^{*} \Gamma_{i} f=$ $\sum_{i \in N} \Gamma_{i}^{*} \Lambda_{i}^{*} f$ holds. All such $g$-frames are called alternate dual $g$-frames of $\left\{\Lambda_{i}: i \in N\right\}$.

Lemma 8. Suppose $\left\{\theta_{i} \in B\left(H, H_{i}\right): i \in N\right\}$ is a $g$ orthonormal basis for $H,\left\{\Lambda_{i} \in B\left(H, H_{i}\right): i \in N\right\}$ is a g-Bessel sequence for $H$, and $V$ and $S$ are the g-preframe operator and g-frame operator associated with $\left\{\Lambda_{i} \in B\left(H, H_{i}\right): i \in N\right\}$, respectively. Then $S=V V^{*}$.

Proof. Since $\Lambda_{i}=\theta_{i} V^{*}$ for all $i \in N$, for any $f \in H$ we have

$$
\begin{aligned}
S f & =\sum_{i \in N} \Lambda_{i}^{*} \Lambda_{i} f=\sum_{i \in N} V \theta_{i}^{*} \theta_{i} V^{*} f \\
& =V \sum_{i \in N} \theta_{i}^{*} \theta_{i} V^{*} f=V V^{*} f .
\end{aligned}
$$

So $S=V V^{*}$. 
Lemma 9 (see, [11]). A sequence $\left\{\theta_{i} \in B\left(H, H_{i}\right): i \in N\right\}$ is a $g$-frame for $H$ if and only if

$$
S: f \longrightarrow \sum_{i \in N} \Lambda_{i}^{*} \Lambda_{i} f
$$

is a well-defined and bounded mapping from $\mathrm{H}$ onto $\mathrm{H}$.

Remark 10. Since $S$ is adjoint, the above condition can be replaced with $S$ is a well-defined and bounded invertible mapping.

Lemma 11 (see, [19]). Suppose that $T: K \rightarrow H$ is a bounded surjective operator. Then there exists a bounded operator (called the pseudoinverse of $T$ ) $T^{\dagger}: H \rightarrow K$ for which

$$
T T^{\dagger} f=f, \quad \forall f \in H .
$$

Theorem 12. Suppose $\left\{\theta_{i} \in B\left(H, H_{i}\right): i \in N\right\}$ is a g-orthonormal basis for $H,\left\{\Lambda_{i} \in B\left(H, H_{i}\right): i \in N\right\}$ is a g-Bessel sequence for $H$, and $V$ and $S$ are the $g$-preframe operator and $g$-frame operator associated with $\left\{\Lambda_{i} \in B\left(H, H_{i}\right): i \in N\right\}$, respectively. Then

(1) $\left\{\Lambda_{i} \in B\left(H, H_{i}\right): i \in N\right\}$ is a $g$-frame if and only if $V$ is onto.

(2) $\left\{\Lambda_{i} \in B\left(H, H_{i}\right): i \in N\right\}$ is a normalized tight $g$-frame if and only if $V$ is a coisometry.

(3) $\left\{\Lambda_{i} \in B\left(H, H_{i}\right): i \in N\right\}$ is a $g$-Riesz basis if and only if $V$ is invertible.

(4) $\left\{\Lambda_{i} \in B\left(H, H_{i}\right): i \in N\right\}$ is a g-orthonormal basis if and only if $V$ is unitary.

Proof. (1) $\Rightarrow$ : By Lemma 9, if $\left\{\Lambda_{i} \in B\left(H, H_{i}\right): i \in N\right\}$ is a $g$-frame, then $S$ is invertible, but $S=V V^{*}$ by Lemma 8 , so $V$ is onto. $\Leftarrow$ : If $V$ is onto, then $\left\{\Lambda_{i} \in B\left(H, H_{i}\right): i \in N\right\}$ is a $g$-Bessel sequence by Lemma 5 . So we just need to show the existence of lower frame bound. Since $V$ is onto, $V V^{\dagger}=I$ by Lemma 11. Hence $\left(V^{\dagger}\right)^{*} V^{*}=I$, so for any $f \in H,\|f\|^{2}=$ $\left\|\left(V^{\dagger}\right)^{*} V^{*} f\right\|^{2} \leq\left\|\left(V^{\dagger}\right)^{*}\right\|^{2}\left\|V^{*} f\right\|^{2}$. So we have

$$
\begin{aligned}
\sum_{i \in N}\left\|\Lambda_{i} f\right\|^{2} & =\sum_{i \in N}\left\|\theta_{i} V^{*} f\right\|^{2} \\
& =\left\|V^{*} f\right\|^{2} \geq \frac{1}{\left\|\left(V^{\dagger}\right)^{*}\right\|^{2}}\|f\|^{2} .
\end{aligned}
$$

So $\left\{\Lambda_{i} \in B\left(H, H_{i}\right): i \in N\right\}$ is a $g$-frame.

(2) Since $\left\{\Lambda_{i} \in B\left(H, H_{i}\right): i \in N\right\}$ is a normalized tight $g$-frame if and only if $S=I$, but $S=V V^{*}$ by Lemma 8 , so $S=I$ if and only $V$ is coisometry.

(3) If $\left\{\Lambda_{i} \in B\left(H, H_{i}\right): i \in N\right\}$ is a $g$-Riesz basis then $V$ is invertible by [11, Corollary 2.18]. Conversely, suppose $V$ is invertible. If for any $i \in N, \Lambda_{i} f=\theta_{i} V^{*} f=0$, since $\left\{\theta_{i}: i \epsilon\right.$ $N\}$ is $g$-complete, then $V^{*} f=0$; hence $f=0$. It follows that $\left\{\Lambda_{i}: i \in N\right\}$ is $g$-complete. Now for any finite set $J \subset N$, and $g_{j} \in H_{j}, j \in J$, we have

$$
\left\|\sum_{j \in J} \Lambda_{j}^{*} g_{j}\right\|^{2}=\left\|\sum_{j \in J} V \theta_{j}^{*} g_{j}\right\|^{2}=\left\|V \sum_{j \in J} \theta_{j}^{*} g_{j}\right\|^{2} .
$$

Since

$$
\begin{gathered}
\left\|\sum_{j \in J} \theta_{j}^{*} g_{j}\right\|^{2}=\left\|V V^{-1} \sum_{j \in J} \theta_{j}^{*} g_{j}\right\|^{2} \leq\left\|V^{-1}\right\|^{2}\left\|V \sum_{j \in J} \theta_{j}^{*} g_{j}\right\|^{2}, \\
\left\|V \sum_{j \in J} \theta_{j}^{*} g_{j}\right\|^{2} \leq\|V\|^{2}\left\|\sum_{j \in J} \theta_{j}^{*} g_{j}\right\|^{2}, \quad\left\|\sum_{j \in J} \theta_{j}^{*} g_{j}\right\|^{2}=\sum_{j \in J}\left\|g_{j}\right\|^{2} .
\end{gathered}
$$

So we have

$$
\frac{1}{\left\|V^{-1}\right\|^{2}} \sum_{j \in J}\left\|g_{j}\right\|^{2} \leq\left\|\sum_{j \in J} \Lambda_{j}^{*} g_{j}\right\|^{2} \leq\|V\|^{2} \sum_{j \in J}\left\|g_{j}\right\|^{2} .
$$

Hence $\left\{\Lambda_{i} \in B\left(H, H_{i}\right): i \in N\right\}$ is a $g$-Riesz basis.

(4) If $\left\{\Lambda_{i} \in B\left(H, H_{i}\right): i \in N\right\}$ is a $g$-orthonormal basis, then $V$ is unitary by [11, Corollary 2.19]. Conversely, suppose that $V$ is unitary. Then for $i, j \in N$, and $g_{i} \in H_{i}, g_{j} \in H_{j}$ we have

$$
\begin{aligned}
&\left\langle\Lambda_{i}^{*} g_{i}, \Lambda_{j}^{*} g_{j}\right\rangle=\left\langle V \theta_{i}^{*} g_{i}, V \theta_{j}^{*} g_{j}\right\rangle \\
&=\left\langle\theta_{i}^{*} g_{i}, \theta_{j}^{*} g_{j}\right\rangle=\delta_{i j}\left\langle g_{i}, g_{j}\right\rangle, \\
& \sum_{i \in N}\left\|\Lambda_{i}^{*} f\right\|^{2}=\sum_{i \in N}\left\|\theta_{i} V^{*} f\right\|^{2}=\left\|V^{*} f\right\|^{2}=\|f\|^{2} .
\end{aligned}
$$

So $\left\{\Lambda_{i} \in B\left(H, H_{i}\right): i \in N\right\}$ is a $g$-orthonormal basis.

Lemma 13. (1) Suppose that $T, V \in B(H)$ and $V$ is onto. Then $T V$ is onto if and only if $T$ is onto.

(2) Suppose that $T, V \in B(H)$ and $V$ is a coisometry. Then $T V$ is a coisometry if and only if $T$ is a coisometry.

(3) Suppose that $T, V \in B(H)$ and $V$ is invertible. Then $T V$ is invertible if and only if $T$ is invertible.

(4) Suppose that $T, V \in B(H)$ and $V$ is a unitary. Then $T V$ is unitary if and only if $T$ is unitary.

Proof. (1) If $T V$ is onto, then for any $y \in H$, there exists $x \in H$ such that $y=T V x=T(V x)$, so $T$ is onto. Conversely, if $T$ is onto, then for any $y \in H$ there exists $x \in H$ such that $y=T x$. Since $V$ is onto, there exists $z \in H$ such that $x=V z$. So $y=T V z$, which implies that $T V$ is onto.

(2) If $T V$ is a coisometry, then $(T V)(T V)^{*}=I$. Since $V$ is a coisometry, $V V^{*}=I$. So $T T^{*}=T\left(V V^{*}\right) T^{*}=(T V)(T V)^{*}=$ $I$, which implies that $T$ is a coisometry. Conversely, if $T$ is a coisometry, then $(T V)(T V)^{*}=T V V^{*} T^{*}=T\left(V V^{*}\right) T^{*}=$ $T T^{*}=I$. Hence $T V$ is a coisometry.

(3) If $T V$ is invertible, then $T=(T V) V^{-1}$, so $T$ is invertible. Conversely, if $T$ is invertible, since $V$ is invertible, then $T V$ is invertible.

(4) If $T V$ is unitary, then $T=T V V^{*}=(T V) V^{*}$, so $T$ is unitary. Conversely, if $T$ is unitary, since $V$ is unitary, so $T V$ is unitary.

By Theorem 12 and Lemma 13, it is easy to get the following results. We leave the details to the readers. 
Theorem 14. Suppose that $\left\{\theta_{i} \in B\left(H, H_{i}\right): i \in N\right\}$ is a $g$ orthonormal basis for $H$ with respect to $\left\{H_{i}: i \in N\right\}, T \in$ $B(H)$. Define the transformation $\Phi_{T}:\left\{\Lambda_{i} \in B\left(H, H_{i}\right): i \in\right.$ $N\} \rightarrow\left\{\Lambda_{i} T^{*}: i \in N\right\}$, then:

(1) It transforms $g$-frames to $g$-frames if and only if $T$ is onto.

(2) Ittransforms normalized tight $g$-frames to normalized tight $g$-frames if and only if $T$ is a coisometry.

(3) Ittransforms $g$-Riesz bases to $g$-Riesz bases if and only if $T$ is invertible.

(4) Ittransforms g-orthonormal bases to g-orthonormal bases if and only if $T$ is unitary.

Remark 15. The above results are established under the constraint that $H$ has a $g$-orthonormal basis with respect to $\left\{H_{i}: i \in N\right\}$. But in general, the statement (1) in the above Theorem is still true.

Theorem 16. Given $T \in B(H)$. The transformation $\Phi_{T}:\left\{\Lambda_{i} \in\right.$ $\left.B\left(H, H_{i}\right): i \in N\right\} \rightarrow\left\{\Lambda_{i} T^{*}: i \in N\right\}$, transforms $g$-frames to $g$-frames if and only if $T$ is onto.

Proof. $\Rightarrow$ : If $\Phi_{T}$ transforms $g$-frames to $g$-frames, then $\left\{\Lambda_{i} T^{*}: i \in N\right\}$ is a $g$-frame whenever $\left\{\Lambda_{i}: i \in N\right\}$ is a $g$-frame. Suppose that $A_{1}, B_{1}$ and $A_{2}, B_{2}$ are the lower and upper frame bounds of $\left\{\Lambda_{i} T^{*}: i \in N\right\}$ and $\left\{\Lambda_{i}: i \in N\right\}$ respectively. Then for any $f \in H$, we have

$$
A_{1}\|f\|^{2} \leq \sum_{i \in N}\left\|\Lambda_{i} T^{*} f\right\|^{2} \leq B_{1}\|f\|^{2} .
$$

So if $T^{*} f=0$, then $f=0$, which implies that $T^{*}$ is one to one. If $T^{*} f_{n} \rightarrow y(n \rightarrow \infty)$, then $\left\{f_{n}\right\}$ is convergent; that is, there exists $y_{0} \in H$ such that $f_{n} \rightarrow y_{0}$. Hence $T^{*} f_{n} \rightarrow T^{*} y_{0}$, so $y=T^{*} y_{0}$, that is, $y \in \operatorname{range}\left(T^{*}\right)$, which implies that $T^{*}$ has closed range. So $T$ has closed range. Since $\operatorname{range}(T)=$ $\overline{\operatorname{range}}(T)=\left(\operatorname{ker} T^{*}\right)^{\perp}=H, T$ is onto.

$\Leftarrow$ : If $T$ is onto and $\left\{\Lambda_{i}: i \in N\right\}$ is a $g$-frame for $H$ with frame bounds $A$ and $B$, then for any $f \in H$, we have

$$
\sum_{i \in N}\left\|\Lambda_{i} T^{*} f\right\|^{2} \leq B\left\|T^{*} f\right\|^{2} \leq B\left\|T^{*}\right\|^{2}\|f\|^{2}
$$

and since $T T^{\dagger}=I$ by Lemma $11,\left(T^{\dagger}\right)^{*} T^{*}=I$. So

$$
\sum_{i \in N}\left\|\Lambda_{i} T^{*} f\right\|^{2} \geq A\left\|T^{*} f\right\|^{2} \geq \frac{A}{\left\|\left(T^{\dagger}\right)^{*}\right\|^{2}}\|f\|^{2} .
$$

So $\left\{\Lambda_{i} T^{*}: i \in N\right\}$ is a $g$-frame for $H$, which implies that $\Phi_{T}$ transforms $g$-frames to $g$-frames.

\section{Sums of $g$-Frames}

In this section, we will consider the direct sums and usual sums of $g$-frames, which generate $g$-frames for direct sum of Hilbert spaces and sum of Hilbert spaces, respectively.

Definition 17. Suppose $T, L \in B(H, K)$, then $T \oplus L \in B(H \oplus$ $H, K)$ is defined by $(T \oplus L)(x \oplus y)=T x+L y$.
Remark 18. Since for any scalars $a$ and $b$, any $x, y \in H$, we have

$$
\begin{aligned}
(T \oplus L) & {\left[a\left(x_{1} \oplus y_{1}\right)+b\left(x_{2} \oplus y_{2}\right)\right] } \\
= & (T \oplus L)\left[\left(a x_{1}+b x_{2}\right) \oplus\left(a y_{1}+b y_{2}\right)\right] \\
= & T\left(a x_{1}+b x_{2}\right)+L\left(a y_{1}+b y_{2}\right) \\
= & a T x_{1}+b T x_{2}+a L y_{1}+b L y_{2} \\
= & a\left(T x_{1}+L y_{1}\right)+b\left(T x_{2}+L y_{2}\right) \\
= & a(T \oplus L)\left(x_{1} \oplus y_{1}\right)+b(T \oplus L)\left(x_{2} \oplus y_{2}\right) .
\end{aligned}
$$

So $T \oplus L$ is linear. Since for any $x, y \in H$, we have

$$
\begin{aligned}
\|(T \oplus L)(x \oplus y)\| & =\|T x+L y\| \leq\|T x\|+\|L y\| \\
& \leq\|T\|\|x\|+\|L\|\|y\| \leq M(\|x\|+\|y\|) \\
& \leq M \sqrt{2} \sqrt{\|x\|^{2}+\|y\|^{2}}=\sqrt{2} M\|x \oplus y\|,
\end{aligned}
$$

where $M=\max \{\|T\|,\|L\|\}$. So $T \oplus L$ is bounded. It is easy to see that $\left(T_{1} \oplus T_{2}\right)^{*} z=T_{1}^{*} z \oplus T_{2}^{*} z$ for any $z \in H_{i}$. In fact, for any $x, y \in H$, we have

$$
\begin{aligned}
\left\langle\left(T_{1} \oplus T_{2}\right)(x \oplus y), z\right\rangle= & \left\langle(x \oplus y),\left(T_{1} \oplus T_{2}\right)^{*} z\right\rangle, \\
\left\langle\left(T_{1} \oplus T_{2}\right)(x \oplus y), z\right\rangle & =\left\langle T_{1} x+T_{2} y, z\right\rangle \\
& =\left\langle T_{1} x, z\right\rangle+\left\langle T_{2} y, z\right\rangle \\
& =\left\langle x, T_{1}^{*} z\right\rangle+\left\langle y, T_{2}^{*} z\right\rangle \\
& =\left\langle x \oplus y, T_{1}^{*} z \oplus T_{2}^{*} z\right\rangle .
\end{aligned}
$$

So $\left(T_{1} \oplus T_{2}\right)^{*} z=T_{1}^{*} z \oplus T_{2}^{*} z$. The finite direct sum of operators have the same meaning.

Definition 19. Let $\left\{\Lambda_{j}: j \in N\right\},\left\{\Gamma_{j}: j \in N\right\}$ be normalized tight $g$-frames for Hilbert space $H$ with respect to $\left\{H_{i}: i \in\right.$ $N\}$, if $\left\{\Lambda_{j} \oplus \Gamma_{j}: j \in N\right\}$ is a normalized tight $g$-frame for $H \oplus H$ with respect to $\left\{H_{i}: i \in N\right\}$, then we call them strongly disjoint. More generally, if $\left\{\Lambda_{j}: j \in N\right\}$ and $\left\{\Gamma_{j}: j \in N\right\}$ are $g$ frames with respect to $\left\{H_{i}: i \in N\right\}$ such that $\left\{\Lambda_{j} \oplus \Gamma_{j}: j \in N\right\}$ is a $g$-frame for $H \oplus H$ with respect to $\left\{H_{i}: i \in N\right\}$, then they are called disjoint.

In general, let $\left\{\Lambda_{1 j}: j \in N\right\}, \ldots,\left\{\Lambda_{k j}: j \in N\right\}$ be normalized tight $g$-frames for Hilbert space $H$, if $\left\{\Lambda_{1 j} \oplus \cdots \oplus\right.$ $\left.\Lambda_{k j}: j \in N\right\}$ is a normalized $g$-frame for $H \oplus H \oplus \cdots \oplus H$, which is the inner direct sum of $k$ copies of $H$, then we call them strongly disjoint. If the $k$ number of sequences are $g$ frames and their inner direct sum is a $g$-frame for the inner direct sum of $k$ copies of $H$, then we call them disjoint.

Definition 20. Let $\left\{\Lambda_{j}: j \in N\right\},\left\{\Gamma_{j}: j \in N\right\}$ be normalized tight $g$-frames for Hilbert space $H$, if $\left\{\Lambda_{j} \oplus \Gamma_{j}: j \in N\right\}$ is a $g$-orthonormal basis of $H \oplus H$, then we call them strongly complementary. 
In general, let $\left\{\Lambda_{1 j}: j \in N\right\}, \ldots,\left\{\Lambda_{k j}: j \in N\right\}$ be normalized tight $g$-frames for Hilbert space $H$, if $\left\{\Lambda_{1 j} \oplus \cdots \oplus\right.$ $\left.\Lambda_{k j}: j \in N\right\}$ is a $g$-orthonormal basis for the inner direct sum of $k$ copies of $H$, then we call them strongly complementary.

In [8], the authors characterize sums of frames by the topological and algebraic properties of $P(H)$, the set of orthogonal projections on $H$. In this section, we always assume that there exists a $g$-orthonormal basis $\left\{\theta_{i}: i \in N\right\}$ for $H$ with respect to $\left\{H_{i}: i \in N\right\}$ and we will characterize all kinds of sums of $g$-frames through the properties of $g$ preframe operators.

Theorem 21. Suppose $\left\{\Lambda_{j}: j \in N\right\},\left\{\Gamma_{j}: j \in N\right\}$ are normalized tight $g$-frames for Hilbert space $H, T_{1}$ and $T_{2}$ are the g-preframe operators associated with $\left\{\Lambda_{j}: j \in N\right\}$ and $\left\{\Gamma_{j}: j \in N\right\}$, respectively; then $\left\{\Lambda_{j}: j \in N\right\}$ and $\left\{\Gamma_{j}: j \in N\right\}$ are strongly disjoint if and only if $T_{2} T_{1}^{*}=0$.

Proof. $\Rightarrow$ : Since $\left\{\Lambda_{j}: j \in N\right\}$ and $\left\{\Gamma_{j}: j \in N\right\}$ are strongly disjoint, so $\left\{\Lambda_{j} \oplus \Gamma_{j}: j \in N\right\}$ is a normalized tight $g$-frame for $H \oplus H$. Since $T_{1}, T_{2}$ are $g$-preframe operators associated with $\left\{\Lambda_{j}: j \in N\right\}$ and $\left\{\Gamma_{j}: j \in N\right\}$, respectively, so $T_{1}$ and $T_{2}$ are coisometries by Theorem 12 and $\Lambda_{n}=\theta_{n} T_{1}^{*}, \Gamma_{n}=\theta_{n} T_{2}^{*}$ for any $n \in N$. So for any $x, y \in H$, we have

$$
\begin{aligned}
\|x\|^{2}+\|y\|^{2} & =\|x \oplus y\|^{2} \\
& =\sum_{i \in N}\left\|\left(\Lambda_{i} \oplus \Gamma_{i}\right)(x \oplus y)\right\|^{2} \\
& =\sum_{i \in N}\left\|\Lambda_{i} x+\Gamma_{i} y\right\|^{2} \\
& =\sum_{i \in N}\left\|\theta_{i} T_{1}^{*} x+\theta_{i} T_{2}^{*} y\right\|^{2} \\
& =\sum_{i \in N}\left\|\theta_{i}\left(T_{1}^{*} x+T_{2}^{*} y\right)\right\|^{2} \\
& =\left\|T_{1}^{*} x+T_{2}^{*} y\right\|^{2} \\
& =\|x\|^{2}+\|y\|^{2}+2 \operatorname{Re}\left\langle T_{2} T_{1}^{*} x, y\right\rangle .
\end{aligned}
$$

So $\operatorname{Re}\left\langle T_{2} T_{1}^{*} x, y\right\rangle=0$. Replace $y$ by $i y$ in the above argument; then we get $\operatorname{Im}\left\langle T_{2} T_{1}^{*} x, y\right\rangle=0$. Hence $\left\langle T_{2} T_{1}^{*} x, y\right\rangle=0$ for any $x, y \in H$. Therefore $T_{2} T_{1}^{*}=0$.

$\Leftarrow$ : Suppose $T_{2} T_{1}^{*}=0$; then for any $x, y \in H$, we have

$$
\begin{aligned}
\sum_{i \in N}\left\|\left(\Lambda_{i} \oplus \Gamma_{i}\right)(x \oplus y)\right\|^{2} & =\sum_{i \in N}\left\|\Lambda_{i} x+\Gamma_{i} y\right\|^{2} \\
& =\sum_{i \in N}\left\|\theta_{i} T_{1}^{*} x+\theta_{i} T_{2}^{*} y\right\|^{2} \\
& =\sum_{i \in N}\left\|\theta_{i}\left(T_{1}^{*} x+T_{2}^{*} y\right)\right\|^{2}
\end{aligned}
$$

$$
\begin{aligned}
= & \left\|T_{1}^{*} x+T_{2}^{*} y\right\|^{2} \\
= & \left\|T_{1}^{*} x\right\|^{2}+\left\|T_{2}^{*} y\right\|^{2} \\
& +2 \operatorname{Re}\left\langle T_{1}^{*} x, T_{2}^{*} y\right\rangle \\
= & \|x\|^{2}+\|y\|^{2}+2 \operatorname{Re}\left\langle T_{2} T_{1}^{*} x, y\right\rangle \\
= & \|x\|^{2}+\|y\|^{2}=\|x \oplus y\|^{2} .
\end{aligned}
$$

So $\left\{\Lambda_{j} \oplus \Gamma_{j}: j \in N\right\}$ is a normalized tight $g$-frame for $H \oplus H$; that is, $\left\{\Lambda_{n}: n \in N\right\}$ and $\left\{\Gamma_{n}: n \in N\right\}$ are strongly disjoint.

It is easy to see that for any finite number of normalized tight $g$-frames, we have the following result.

Corollary 22. Suppose $\left\{\Lambda_{1 j}: j \in N\right\}, \ldots,\left\{\Lambda_{k j}: j \in N\right\}$ are normalized tight $g$-frames for Hilbert space $H, T_{1}, \ldots, T_{k}$ are g-preframe operators associated with $\left\{\Lambda_{1 j}: j \in N\right\}, \ldots,\left\{\Lambda_{k j}\right.$ : $j \in N\}$, respectively; then $\left\{\Lambda_{1 j}: j \in N\right\}, \ldots,\left\{\Lambda_{k j}: j \in N\right\}$ are strongly disjoint if and only if $T_{m} T_{n}^{*}=0, m, n=1, \ldots, k$.

Theorem 23. Suppose $\left\{\Lambda_{j}: j \in N\right\}$ and $\left\{\Gamma_{j}: j \in N\right\}$ are g-frames for Hilbert space $H$ with respect to $\left\{H_{i}: i \in N\right\}, T_{1}$ and $T_{2}$ are g-preframe operators associated with $\left\{\Lambda_{j}: j \in N\right\}$ and $\left\{\Gamma_{j}: j \in N\right\}$, respectively. If $T_{2} T_{1}^{*}=0$, then $\left\{\Lambda_{j}: j \in N\right\}$ and $\left\{\Gamma_{j}: j \in N\right\}$ are disjoint.

Proof. Since $T_{1}$ and $T_{2}$ are $g$-preframe operators associated with $g$-frames $\left\{\Lambda_{j}: j \in N\right\}$ and $\left\{\Gamma_{j}: j \in N\right\}$, respectively, so $T_{1}$ and $T_{2}$ are onto operators by Theorem 12 . So there exist $T_{1}^{\dagger}$ and $T_{2}^{\dagger}$ such that $T_{1} T_{1}^{\dagger}=I$ and $T_{2} T_{2}^{\dagger}=I$ by Lemma 11. So $\left(T_{1}^{\dagger}\right)^{*} T_{1}^{*}=I$ and $\left(T_{2}^{\dagger}\right)^{*} T_{2}^{*}=I$. Hence $\|x\|=$ $\left\|\left(T_{1}^{\dagger}\right)^{*} T_{1}^{*} x\right\| \leq\left\|\left(T_{1}^{\dagger}\right)^{*}\right\|\left\|T_{1}^{*} x\right\|$, so $\left\|T_{1}^{*} x\right\| \geq\left(1 /\left\|\left(T_{1}^{\dagger}\right)^{*}\right\|\right)\|x\|$. Similarly, $\left\|T_{2}^{*} x\right\| \geq\left(1 /\left\|\left(T_{2}^{\dagger}\right)^{*}\right\|\right)\|x\|$. Since $T_{2} T_{1}^{*}=0$, we have

$$
\begin{aligned}
\sum_{i \in N}\left\|\left(\Lambda_{i} \oplus \Gamma_{i}\right)(x \oplus y)\right\|^{2}= & \sum_{i \in N}\left\|\Lambda_{i} x+\Gamma_{i} y\right\|^{2} \\
= & \sum_{i \in N}\left\|\theta_{i} T_{1}^{*} x+\theta_{i} T_{2}^{*} y\right\|^{2} \\
= & \sum_{i \in N}\left\|\theta_{i}\left(T_{1}^{*} x+T_{2}^{*} y\right)\right\|^{2} \\
= & \left\|T_{1}^{*} x+T_{2}^{*} y\right\|^{2} \\
= & \left\|T_{1}^{*} x\right\|^{2}+\left\|T_{2}^{*} y\right\|^{2} \\
& +2 \operatorname{Re}\left\langle T_{1}^{*} x, T_{2}^{*} y\right\rangle \\
= & \left\|T_{1}^{*} x\right\|^{2}+\left\|T_{2}^{*} y\right\|^{2} \\
& +2 \operatorname{Re}\left\langle T_{2} T_{1}^{*} x, y\right\rangle \\
= & \left\|T_{1}^{*} x\right\|^{2}+\left\|T_{2}^{*} y\right\|^{2} .
\end{aligned}
$$


Since

$$
\begin{aligned}
\left\|T_{1}^{*} x\right\|^{2}+\left\|T_{2}^{*} y\right\|^{2} & \leq\left\|T_{1}^{*}\right\|^{2} \cdot\|x\|^{2}+\left\|T_{2}^{*}\right\|^{2} \cdot\|x\|^{2} \\
& \leq B \cdot\left(\|x\|^{2}+\|y\|^{2}\right)=B \cdot\|x \oplus y\|^{2},
\end{aligned}
$$

where $B=\max \left\{\left\|T_{1}^{*}\right\|^{2},\left\|T_{2}^{*}\right\|^{2}\right\}$. Hence $\sum_{i \in N} \|\left(\Lambda_{i} \oplus \Gamma_{i}\right)$ $(x \oplus y)\left\|^{2} \leq B \cdot\right\| x \oplus y \|^{2}$. Since

$$
\begin{aligned}
\left\|T_{1}^{*} x\right\|^{2}+\left\|T_{2}^{*} y\right\|^{2} & \geq \frac{1}{\left\|\left(T_{1}^{\dagger}\right)^{*}\right\|^{2}}\|x\|^{2}+\frac{1}{\left\|\left(T_{2}^{\dagger}\right)^{*}\right\|^{2}}\|y\|^{2} \\
& \geq A \cdot\left(\|x\|^{2}+\|y\|^{2}\right)=A \cdot\|x \oplus y\|^{2},
\end{aligned}
$$

where $A=\min \left\{1 /\left\|\left(T_{1}^{\dagger}\right)^{*}\right\|^{2}, 1 /\left\|\left(T_{2}^{\dagger}\right)^{*}\right\|^{2}\right\}$. Hence

$$
\sum_{i \in N}\left\|\left(\Lambda_{i} \oplus \Gamma_{i}\right)(x \oplus y)\right\|^{2} \geq A \cdot\|x \oplus y\|^{2}
$$

So $\left\{\Lambda_{n} \oplus \Gamma_{n}: n \in N\right\}$ is a $g$-frame for $H \oplus H$; that is, $\left\{\Lambda_{j}: j \in\right.$ $N\}$ and $\left\{\Gamma_{j}: j \in N\right\}$ are disjoint.

Similarly, for any finite number of $g$-frames, we have the following.

Corollary 24. Suppose $\left\{\Lambda_{1 j}: j \in N\right\}, \ldots,\left\{\Lambda_{k j}: j \in N\right\}$ are $g$-frames for Hilbert space $H, T_{1}, \ldots, T_{k}$ are $g$-preframe operators of $\left\{\Lambda_{1 j}: j \in N\right\}, \ldots,\left\{\Lambda_{k j}: j \in N\right\}$, respectively; If $T_{m} T_{n}^{*}=0, m, n=1, \ldots, k$, then $\left\{\Lambda_{1 j}: j \in N\right\}, \ldots,\left\{\Lambda_{k j}\right.$ : $j \in N\}$ are disjoint.

Theorem 25. Let $\left\{\Lambda_{n}: n \in N\right\}$ and $\left\{\Gamma_{n}: n \in N\right\}$ be normalized tight $g$-frames for $H, T_{1}$ and $T_{2}$ are $g$-preframe operators associated with $\left\{\Lambda_{n}: n \in N\right\}$ and $\left\{\Gamma_{n}: n \in N\right\}$, respectively. If $\left\{\Lambda_{n}: n \in N\right\}$ and $\left\{\Gamma_{n}: n \in N\right\}$ are strongly complementary, then $T_{2} T_{1}^{*}=0$ and $T_{1}^{*} T_{1}+T_{2}^{*} T_{2}=I$.

Proof. If $\left\{\Lambda_{n}: n \in N\right\}$ and $\left\{\Gamma_{n}: n \in N\right\}$ are strongly complementary, then $\left\{\Lambda_{n} \oplus \Gamma_{n}: n \in N\right\}$ is a $g$-orthonormal basis for $H \oplus H$. So they are of course strongly disjoint, therefore $T_{2} T_{1}^{*}=0$ by Theorem 21. Since $\left\{\theta_{i}: i \in N\right\}$ is a $g$-orthonormal basis for $H$, for any $n, k \in N$ and $g_{n} \in H_{n}$, $g_{k} \in H_{k}$, we have

$$
\begin{aligned}
& \left\langle\left(\Lambda_{n} \oplus \Gamma_{n}\right)^{*} g_{n},\left(\Lambda_{k} \oplus \Gamma_{k}\right)^{*} g_{k}\right\rangle \\
& =\delta_{n k}\left\langle g_{n}, g_{k}\right\rangle=\left\langle\theta_{n}^{*} g_{n}, \theta_{k}^{*} g_{k}\right\rangle .
\end{aligned}
$$

But

$$
\begin{aligned}
& \left\langle\left(\Lambda_{n} \oplus \Gamma_{n}\right)^{*} g_{n},\left(\Lambda_{k} \oplus \Gamma_{k}\right)^{*} g_{k}\right\rangle \\
& =\left\langle\Lambda_{n}^{*} g_{n} \oplus \Gamma_{n}^{*} g_{n}, \Lambda_{k}^{*} g_{k} \oplus \Gamma_{k}^{*} g_{k}\right\rangle
\end{aligned}
$$

$$
\begin{aligned}
& =\left\langle T_{1} \theta_{n}^{*} g_{n} \oplus T_{2} \theta_{n}^{*} g_{n}, T_{1} \theta_{k}^{*} g_{k} \oplus T_{2} \theta_{k}^{*} g_{k}\right\rangle \\
& =\left\langle T_{1} \theta_{n}^{*} g_{n}, T_{1} \theta_{k}^{*} g_{k}\right\rangle+\left\langle T_{2} \theta_{n}^{*} g_{n}, T_{2} \theta_{k}^{*} g_{k}\right\rangle \\
& =\left\langle T_{1}^{*} T_{1} \theta_{n}^{*} g_{n}, \theta_{k}^{*} g_{k}\right\rangle+\left\langle T_{2}^{*} T_{2} \theta_{n}^{*} g_{n}, \theta_{k}^{*} g_{k}\right\rangle \\
& =\left\langle\left(T_{1}^{*} T_{1}+T_{2}^{*} T_{2}\right) \theta_{n}^{*} g_{n}, \theta_{k}^{*} g_{k}\right\rangle .
\end{aligned}
$$

Since $\overline{\operatorname{span}}\left\{\theta_{i}^{*} g_{i}: i \in N\right\}=H$, we have that $T_{1}^{*} T_{1}+T_{2}^{*} T_{2}=$ I.

Similarly, for any finite number of normalized tight $g$ frames, we have

Corollary 26. Suppose $\left\{\Lambda_{1 j}: j \in N\right\}, \ldots,\left\{\Lambda_{k j}: j \in N\right\}$ are normalized tight $g$-frames for Hilbert space $H, T_{1}, \ldots, T_{k}$ are g-preframe operators associated with $\left\{\Lambda_{1 j}: j \in N\right\}, \ldots,\left\{\Lambda_{k j}\right.$ : $j \in N\}$, respectively, if $\left\{\Lambda_{1 j}: j \in N\right\}, \ldots,\left\{\Lambda_{k j}: j \in N\right\}$ are strongly complementary, then $T_{m} T_{n}^{*}=0, m, n=1, \ldots, k$, and $\sum_{i=1}^{k} T_{i}^{*} T_{i}=I$.

The above theorems are related with the inner direct sums of $g$-frames or normalized tight $g$-frames. In the following, we will consider the usual sums of $g$-frames or normalized tight $g$-frames.

Theorem 27. Suppose that $\left\{\Lambda_{n}: n \in N\right\}$ and $\left\{\Gamma_{n}: n \in N\right\}$ are $g$-frames for Hilbert space $H, T_{1}$ and $T_{2}$ are g-preframe operators associated with $\left\{\Lambda_{n}: n \in N\right\}$ and $\left\{\Gamma_{n}: n \in N\right\}$, respectively. If $T_{2} T_{1}^{*}=0$, then $\left\{\Lambda_{n}+\Gamma_{n}: n \in N\right\}$ is a $g$-frame for $H$. Moreover, if $\left\{\Lambda_{n}: n \in N\right\}$ and $\left\{\Gamma_{n}: n \in N\right\}$ are normalized tight $g$-frames and $T_{2} T_{1}^{*}=0$, then $\left\{\Lambda_{n}+\Gamma_{n}: n \in N\right\}$ is a tight $g$-frame for $H$ with bounds 2 .

Proof. Since $T_{1}$ and $T_{2}$ are $g$-preframe operators associated with $g$-frames $\left\{\Lambda_{n}: n \in N\right\}$ and $\left\{\Gamma_{n}: n \in N\right\}$, respectively, so $\Lambda_{n}=\theta_{n} T_{1}^{*}$ and $\Gamma_{n}=\theta_{n} T_{2}^{*}$ for any $n \in N$, where $\left\{\theta_{n}: n \in N\right\}$ is a $g$-orthonormal basis for $H$. Hence $\Lambda_{n}+\Gamma_{n}=\theta_{n}\left(T_{1}+T_{2}\right)^{*}$ for any $n \in N$. To show $\left\{\Lambda_{n}+\Gamma_{n}: n \in N\right\}$ is a $g$-frame, it is sufficient to show that $T_{1}+T_{2}$ is onto by Theorem 12 . Since $T_{2} T_{1}^{*}=0$, so

$$
\left(T_{1}+T_{2}\right) T_{1}^{*}=T_{1} T_{1}^{*}+T_{2} T_{1}^{*}=T_{1} T_{1}^{*} .
$$

Since $T_{1} T_{1}^{*}$ is invertible, so for any $z \in H$, there exists $x=$ $T_{1}^{*}\left(T_{1} T_{1}^{*}\right)^{-1} z \in H$ such that

$$
\begin{aligned}
\left(T_{1}+T_{2}\right) x & =\left(T_{1}+T_{2}\right) T_{1}^{*}\left(T_{1} T_{1}^{*}\right)^{-1} z \\
& =\left(T_{1} T_{1}^{*}\right)\left(T_{1} T_{1}^{*}\right)^{-1} z=z .
\end{aligned}
$$

So $T_{1}+T_{2}$ is onto.

If $\left\{\Lambda_{n}: n \in N\right\}$ and $\left\{\Gamma_{n}: n \in N\right\}$ are normalized tight $g$-frames and $T_{2} T_{1}^{*}=0$, then $\left\{\Lambda_{n}+\Gamma_{n}: n \in N\right\}$ is a $g$-frame and for any $x \in H$, we have

$$
\begin{aligned}
& \sum_{n \in N}\left\|\left(\Lambda_{n}+\Gamma_{n}\right) x\right\|^{2} \\
&=\sum_{n \in N}\left\|\Lambda_{n} x\right\|^{2}+\sum_{n \in N}\left\|\Gamma_{n} x\right\|^{2} \\
&+\sum_{n \in N}\left\langle\Lambda_{n} x, \Gamma_{n} x\right\rangle+\sum_{n \in N}\left\langle\Gamma_{n} x, \Lambda_{n} x\right\rangle
\end{aligned}
$$




$$
\begin{aligned}
= & 2\|x\|^{2}+\sum_{n \in N}\left\langle\theta_{n} T_{1}^{*} x, \theta_{n} T_{2}^{*} x\right\rangle \\
& +\sum_{n \in N}\left\langle\theta_{n} T_{2}^{*} x, \theta_{n} T_{1}^{*} x\right\rangle \\
= & 2\|x\|^{2}+\sum_{n \in N}\left\langle T_{2} \theta_{n}^{*} \theta_{n} T_{1}^{*} x, x\right\rangle \\
& +\sum_{n \in N}\left\langle T_{1} \theta_{n}^{*} \theta_{n} T_{2}^{*} x, x\right\rangle \\
= & 2\|x\|^{2}+\left\langle T_{2} \sum_{n \in N} \theta_{n}^{*} \theta_{n} T_{1}^{*} x, x\right\rangle \\
& +\left\langle T_{1} \sum_{n \in N} \theta_{n}^{*} \theta_{n} T_{2}^{*} x, x\right\rangle \\
= & 2\|x\|^{2}+\left\langle T_{2} T_{1}^{*} x, x\right\rangle+\left\langle T_{1} T_{2}^{*} x, x\right\rangle \\
= & 2\|x\|^{2} .
\end{aligned}
$$

So $\left\{\Lambda_{n}+\Gamma_{n}: n \in N\right\}$ is a tight $g$-frame with bound 2 .

Similarly, for any finite number of $g$-frames, we have the following.

Corollary 28. Suppose $\left\{\Lambda_{1 j}: j \in N\right\}, \ldots,\left\{\Lambda_{k j}: j \in N\right\}$ are $g$-frames for Hilbert space $H, T_{1}, \cdots, T_{k}$ are $g$-preframe operators associated with $\left\{\Lambda_{1 j}: j \in N\right\}, \ldots,\left\{\Lambda_{k j}: j \in N\right\}$, respectively. If $T_{m} T_{n}^{*}=0, m, n=1, \ldots, k$, then $\left\{\Lambda_{1 j}+\cdots+\right.$ $\left.\Lambda_{k j}: j \in N\right\}$ is a $g$-frame for $H$. Moreover, if $\left\{\Lambda_{1 j}\right.$ : $j \in N\}, \ldots,\left\{\Lambda_{k j}: j \in N\right\}$ are normalized tight $g$-frames for Hilbert space $H$ and $T_{m} T_{n}^{*}=0, m, n=1, \ldots, k$, then $\left\{\Lambda_{1 j}+\cdots+\Lambda_{k j}: j \in N\right\}$ is a tight $g$-frame for $H$ with bound $k$.

The above theorem can be generalized further as follows.

Theorem 29. Suppose that $\left\{\Lambda_{n}: n \in N\right\}$ and $\left\{\Gamma_{n}: n \in N\right\}$ are $g$-frames for Hilbert space $H, T_{1}$ and $T_{2}$ are $g$-preframe operators associated with $\left\{\Lambda_{n}: n \in N\right\}$ and $\left\{\Gamma_{n}: n \in N\right\}$, respectively, such that $T_{2} T_{1}^{*}=0$ and $A, B \in B(H)$. If either $A$ or $B$ is onto, then $\left\{\Lambda_{n} A^{*}+\Gamma_{n} B^{*}: n \in N\right\}$ is a $g$-frame for $H$.

Proof. Since $T_{1}$ and $T_{2}$ are $g$-preframe operators associated with $\left\{\Lambda_{n}: n \in N\right\}$ and $\left\{\Gamma_{n}: n \in N\right\}$, respectively, $\Lambda_{n}=\theta_{n} T_{1}^{*}$ and $\Gamma_{n}=\theta_{n} T_{2}^{*}$. So

$$
\begin{aligned}
\Lambda_{n} A^{*} & +\Gamma_{n} B^{*}=\theta_{n} T_{1}^{*} A^{*}+\theta_{n} T_{2}^{*} B^{*} \\
& =\theta_{n}\left(T_{1}^{*} A^{*}+T_{2}^{*} B^{*}\right)=\theta_{n}\left(A T_{1}+B T_{2}\right)^{*} .
\end{aligned}
$$

To show $\left\{\Lambda_{n} A^{*}+\Gamma_{n} B^{*}: n \in N\right\}$ is a $g$-frame it is sufficient to show that $A T_{1}+B T_{2}$ is onto by Theorem 12 . It is well known that $T_{1} T_{1}^{*}$ is invertible. If $A$ is onto, then for any $z \in H$, there exists $x \in H$ such that $A x=z$. So for any $z \in H$, there exists $f=T_{1}^{*}\left(T_{1} T_{1}^{*}\right)^{-1} x \in H$ such that

$$
\begin{aligned}
\left(A T_{1}+B T_{2}\right) f & =\left(A T_{1}+B T_{2}\right) T_{1}^{*}\left(T_{1} T_{1}^{*}\right)^{-1} x \\
& =A T_{1} T_{1}^{*}\left(T_{1} T_{1}^{*}\right)^{-1} x=A x=z .
\end{aligned}
$$

It follows that $A T_{1}+B T_{2}$ is onto. In the case that $B$ is onto, the result can be proved similarly.

Similarly, for any finite number of $g$-frames, we have the following.

Corollary 30. Suppose $\left\{\Lambda_{1 j}: j \in N\right\}, \ldots,\left\{\Lambda_{k j}: j \in N\right\}$ are g-frames for Hilbert space $H$, and $T_{1}, \ldots, T_{k}$ are g-preframe operators associated with $\left\{\Lambda_{1 j}: j \in N\right\}, \ldots,\left\{\Lambda_{k j}: j \in N\right\}$, respectively, and $T_{n} T_{m}^{*}=0, m, n=1,2, \ldots, k, A_{i} \in B(H), i=$ $1, \ldots, k$. If there exists $i \in\{1,2, \ldots, k\}$ such that $A_{i}$ is surjective, then $\left\{\sum_{i=1}^{k} \Lambda_{i j} A_{i}^{*}\right\}$ is a $g$-frame for $H$.

\section{Dual $g$-Frames of $g$-Frames}

In this section, we study the dual $g$-frames by using the properties of $g$-preframe operators.

Theorem 31. Suppose g-frames $\left\{\Lambda_{n}: n \in N\right\}$ and $\left\{\Gamma_{n}: n \in\right.$ $N\}$ are alternate dual $g$-frame pair for Hilbert space $H, T$ is a coisometry in $B(H)$. Then $\left\{\Lambda_{n} T^{*}\right\}$ and $\left\{\Gamma_{n} T^{*}: n \in N\right\}$ are alternate dual $g$-frame pair for $H$.

Proof. Since $\left\{\Lambda_{n}: n \in N\right\}$ and $\left\{\Gamma_{n}: n \in N\right\}$ are $g$-frames for $H$ and $T$ is a coisometry, so $\left\{\Lambda_{n} T^{*}\right\}$ and $\left\{\Gamma_{n} T^{*}: n \in N\right\}$ are also $g$-frames for $H$ by Theorem 14 . Since $\left\{\Lambda_{n}: n \in N\right\}$ and $\left\{\Gamma_{n}: n \in N\right\}$ are alternate dual $g$-frame pair, we have, for any $x \in H$

$$
x=\sum_{n \in N} \Lambda_{n}^{*} \Gamma_{n} x .
$$

So

$$
T x=\sum_{n \in N} T \Lambda_{n}^{*} \Gamma_{n} x=\sum_{n \in N}\left(\Lambda_{n} T^{*}\right)^{*} \Gamma_{n} x .
$$

Replacing $x$ by $T^{*} x$ and applying $T T^{*}=I$, we have

$$
x=T T^{*} x=\sum_{n \in N}\left(\Lambda_{n} T^{*}\right)^{*} \Gamma_{n} T^{*} x .
$$

So $\left\{\Lambda_{n} T^{*}\right\}$ and $\left\{\Gamma_{n} T^{*}: n \in N\right\}$ are alternate dual $g$-frame pair for $H$.

More general, we have the following result.

Theorem 32. Suppose $\left\{\Lambda_{n}: n \in N\right\}$ and $\left\{\Gamma_{n}: n \in N\right\}$ are alternate dual $g$-frame pair for Hilbert space $H, T$ is a surjective operator in $B(H)$. Then $\left\{\Lambda_{n} T^{*}: n \in N\right\}$ and $\left\{\Gamma_{n} T^{\dagger}: n \in N\right\}$ are alternate dual $g$-frame pair for $H$. Similarly, $\left\{\Lambda_{n} T^{\dagger}\right\}$ and $\left\{\Gamma_{n} T^{*}: n \in N\right\}$ are alternate dual $g$-frame pair for $H$. 
Proof. Since $T$ is onto, $T T^{\dagger}=I$ by Lemma 11, so $\left(T^{\dagger}\right)^{*} T^{*}=I$. It follows that $\left(T^{\dagger}\right)^{*}$ is onto. Since $\left\{\Lambda_{n}: n \in N\right\}$ and $\left\{\Gamma_{n}: n \in\right.$ $N\}$ are $g$-frames for $H$, so $\left\{\Lambda_{n} T^{*}: n \in N\right\},\left\{\Gamma_{n} T^{*}: n \in N\right\}$, $\left\{\Lambda_{n} T^{\dagger}: n \in N\right\}$ and $\left\{\Gamma_{n} T^{\dagger}: n \in N\right\}$ are $g$-frames for $H$ by Theorem 14. Since $\left\{\Lambda_{n}: n \in N\right\}$ and $\left\{\Gamma_{n}: n \in N\right\}$ are alternate dual $g$-frame pair, we have, for any $x \in H$

$$
x=\sum_{n \in N} \Lambda_{n}^{*} \Gamma_{n} x
$$

So

$$
T x=\sum_{n \in N} T \Lambda_{n}^{*} \Gamma_{n} x=\sum_{n \in N}\left(\Lambda_{n} T^{*}\right)^{*} \Gamma_{n} x
$$

Replaced $x$ by $T^{\dagger} x$ and applying $T T^{\dagger}=I$, we have

$$
x=T T^{\dagger} x=\sum_{n \in N}\left(\Lambda_{n} T^{*}\right)^{*} \Gamma_{n} T^{\dagger} x
$$

So $\left\{\Lambda_{n} T^{*}\right\}$ and $\left\{\Gamma_{n} T^{\dagger}: n \in N\right\}$ are alternate dual $g$-frame pair for $H$. Interchanging $\Lambda_{n}$ and $\Gamma_{n}$ for any $n \in N$, we get that $\left\{\Lambda_{n} T^{\dagger}\right\}$ and $\left\{\Gamma_{n} T^{*}: n \in N\right\}$ are alternate dual $g$-frame pair for $H$.

Theorem 33. Let $\left\{\Lambda_{n}: n \in N\right\}$ be a g-frame for $H$. Let $\left\{\widetilde{\Lambda}_{n}\right.$ : $n \in N\}$ denote the canonical dual $g$-frame of $\left\{\Lambda_{n}: n \in N\right\}$ and $T_{x}$ denote the the g-preframe operator associated with $\left\{\Lambda_{n}\right.$ : $n \in N\}$. If $P$ is an orthogonal projection with $P T_{x}=T_{x} P$, then $\left\{\Lambda_{n} P: n \in N\right\}$ is a $g$-frame for $P H$ and the canonical dual g-frame for $\left\{\Lambda_{n} P: n \in N\right\}$ is $\left\{\widetilde{\Lambda}_{n} P: n \in N\right\}$.

Proof. It is easy to see that $\left\{\Lambda_{n} P: n \in N\right\}$ is a $g$-frame for $P H$ and $\left\{\theta_{n} P: n \in N\right\}$ is a $g$-orthonormal basis for $P H$ with respect to $\left\{H_{i}: n \in N\right\}$. We only need to prove the second part. By definition, the canonical dual $g$-frame for $\left\{\Lambda_{n}: n \in N\right\}$ is $\left\{\Lambda_{n} S^{-1}: n \in N\right\}$, where $S=T_{x} T_{x}^{*}$. So $\widetilde{\Lambda}_{n}=\Lambda_{n} S^{-1}$. Since $P T_{x}=T_{x} P$, so $P T_{x}^{*}=T_{x}^{*} P$. Since $\Lambda_{n}=$ $\theta_{n} T_{x}^{*}$, so $\Lambda_{n} P=\theta_{n} T_{x}^{*} P=\theta_{n} P T_{x}^{*}$. Hence the $g$-preframe operator associated with $\left\{\Lambda_{n} P: n \in N\right\}$ is $T_{x}$ as well. So the canonical dual $g$-frame for $\left\{\Lambda_{n} P\right\}$ is $\left\{\Lambda_{n} P\left(T_{x} T_{x}^{*}\right)^{-1}=\right.$ $\left\{\Lambda_{n}\left(T_{x} T_{x}^{*}\right)^{-1} P\right\}=\left\{\widetilde{\Lambda}_{n} P: n \in N\right\}$.

The following theorem characterizes the alternate dual $g$ frames in terms of $g$-preframe operators.

Theorem 34. Let $\left\{\Lambda_{n}: n \in N\right\}$ and $\left\{\Gamma_{n}: n \in N\right\}$ be $g$ frames for Hilbert space $H$, and let $T_{x}$ and $T_{y}$ be the g-preframe operators associated with $\left\{\Lambda_{n}: n \in N\right\}$ and $\left\{\Gamma_{n}: n \in N\right\}$, respectively. Then $\left\{\Lambda_{n}: n \in N\right\}$ and $\left\{\Gamma_{n}: n \in N\right\}$ are alternate dual $g$-frame pair if and only if $T_{y} T_{x}^{*}=I$ or $T_{x} T_{y}^{*}=I$.

Proof. Since $T_{x}$ and $T_{y}$ are $g$-preframe operators associated with $\left\{\Lambda_{n}: n \in N\right\}$ and $\left\{\Gamma_{n}: n \in N\right\}$, respectively, so $\theta_{n} T_{x}^{*}=$ $\Lambda_{n}$ and $\theta_{n} T_{y}^{*}=\Gamma_{n}$ for any $n \in N$. Hence for any $x \in H$,

$$
\sum_{n \in N} \Lambda_{n}^{*} \Gamma_{n} x=\sum_{n \in N} T_{x} \theta_{n}^{*} \theta_{n} T_{y}^{*} x=T_{x} T_{y}^{*} x
$$

Similarly, $\sum_{n \in N} \Gamma_{n}^{*} \Lambda_{n} x=T_{y} T_{x}^{*} x$. It follows that $\left\{\Lambda_{n}: n \in N\right.$ and $\left\{\Gamma_{n}: n \in N\right\}$ are alternate dual $g$-frame pair if and only if $T_{y} T_{x}^{*}=I$ or $T_{x} T_{y}^{*}=I$.
Remark 35. Throughout this paper we assume that there exists $g$-orthonormal basis for Hilbert space $H$. It is well known that any Hilbert space has orthonormal basis. A natural question is that given Hilbert space $H, K$ and sequence of Hilbert space $\left\{H_{i}: i \in J\right\} \subset K$, Do the $g$-orthonormal bases for $H$ with respect to $\left\{H_{i}: i \in J\right\}$ always exist? if not, what is the condition such that the $g$-orthonormal bases exist? These interesting questions will be our next research subjects.

\section{Acknowledgment}

This work was partially supported by SWUFE's Key Subjects Construction Items Funds of 211 Project.

\section{References}

[1] R. J. Duffin and A. C. Shaffer, "A class of nonharmonic Fourier series," Transactions of the American Mathematical Society, vol. 72, pp. 341-366, 1952.

[2] B. Sz-Nagy, "Expansion theorems of Paley-Wiener type," Duke Mathematical Journal, vol. 14, pp. 975-978, 1947.

[3] R. M. Young, An Introduction to Nonharmonic Fourier Series, Academic Press, New York, NY, USA, 1980.

[4] C. K. Chui, An Introduction to Wavelets, Academic Press, New York, NY, USA, 1992.

[5] I. Daubechies, Ten Lectures on Wavelets, vol. 61 of CBMS-NSF Regional Conference Series in Applied Mathematics, Society for Industrial and Applied Mathematics (SIAM), 1992.

[6] E. Hernández and G. Weiss, A First Course on Wavelets, CRC Press, Boca Raton, Fla, USA, 1996.

[7] S. G. Mallat, "Multiresolution approximations and wavelet orthonormal bases of $\mathrm{L}^{2}(\mathrm{R})$," Transactions of the American Mathematical Society, vol. 315, no. 1, pp. 69-87, 1989.

[8] D. Han and D. Larson, Bases, Frames and Group Representations, vol. 147 of Memoirs of the American Mathematical Society, no. $697,2000$.

[9] W. Sun, “G-frames and g-Riesz bases," Journal of Mathematical Analysis and Applications, vol. 322, no. 1, pp. 437-452, 2006.

[10] Y. Can Zhu, "Characterizations of g-frames and g-Riesz bases in Hilbert spaces," Acta Mathematica Sinica, vol. 24, no. 10, pp. 1727-1736, 2008.

[11] A. Najati, M. H. Faroughi, and A. Rahimi, "G-frames and stability of g-frames in Hilbert spaces," Methods of Functional Analysis and Topology, vol. 14, no. 3, pp. 271-286, 2008.

[12] Y. Jin Wang and Y. Can Zhu, "G-frames and g-frame sequences in Hilbert spaces," Acta Mathematica Sinica, vol. 25, no. 12, pp. 2093-2106, 2009.

[13] A. Khosravi and K. Musazadeh, "Fusion frames and g-frames," Journal of Mathematical Analysis and Applications, vol. 342, no. 2, pp. 1068-1083, 2008.

[14] M. Ling Ding and Y. Can Zhu, "g-Besselian frames in Hilbert spaces," Acta Mathematica Sinica, vol. 26, no. 11, pp. 2117-2130, 2010.

[15] A. Abdollahi and E. Rahimi, "Some results on g-frames in Hilbert spaces," Turkish Journal of Mathematics, vol. 35, no. 4, pp. 695-704, 2011.

[16] J. R. Holub, "On a property of bases in a Hilbert space," Glasgow Mathematical Journal, vol. 46, no. 1, pp. 177-180, 2004. 
[17] S. Obeidat, S. Samarah, P. G. Casazza, and J. C. Tremain, "Sums of Hilbert space frames," Journal of Mathematical Analysis and Applications, vol. 351, no. 2, pp. 579-585, 2009.

[18] P. G. Casazza, "Every frame is a sum of three (but not two) orthonormal bases-and other frame representations," The Journal of Fourier Analysis and Applications, vol. 4, no. 6, pp. 727732, 1998.

[19] O. Christensen and T. K. Jensen, An Introduction to the Theory of Bases, Frames, and Wavelets, Technical University of Denmark, 1999. 


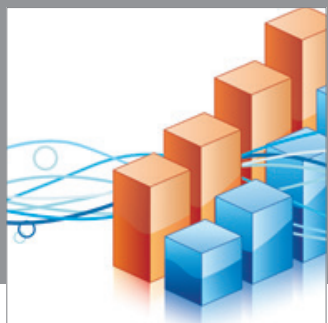

Advances in

Operations Research

mansans

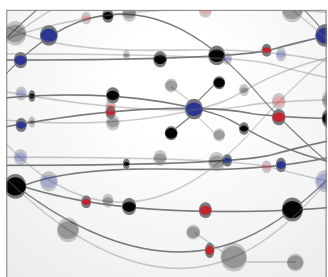

The Scientific World Journal
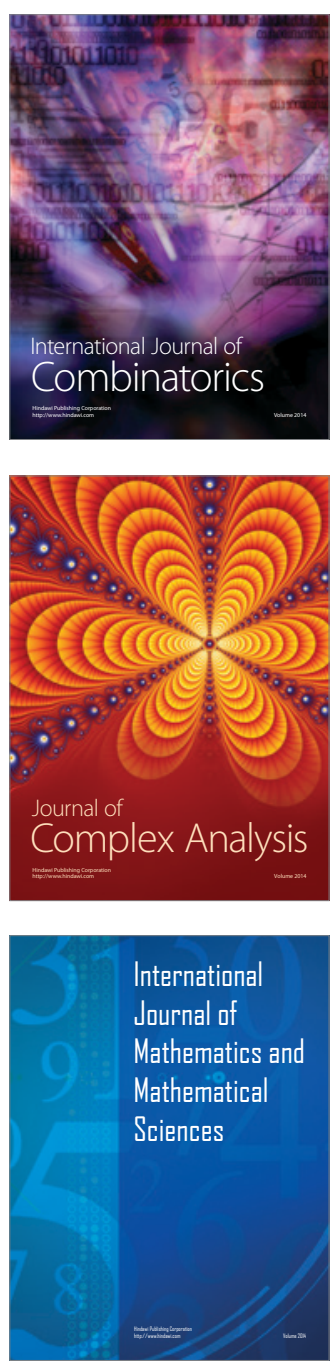
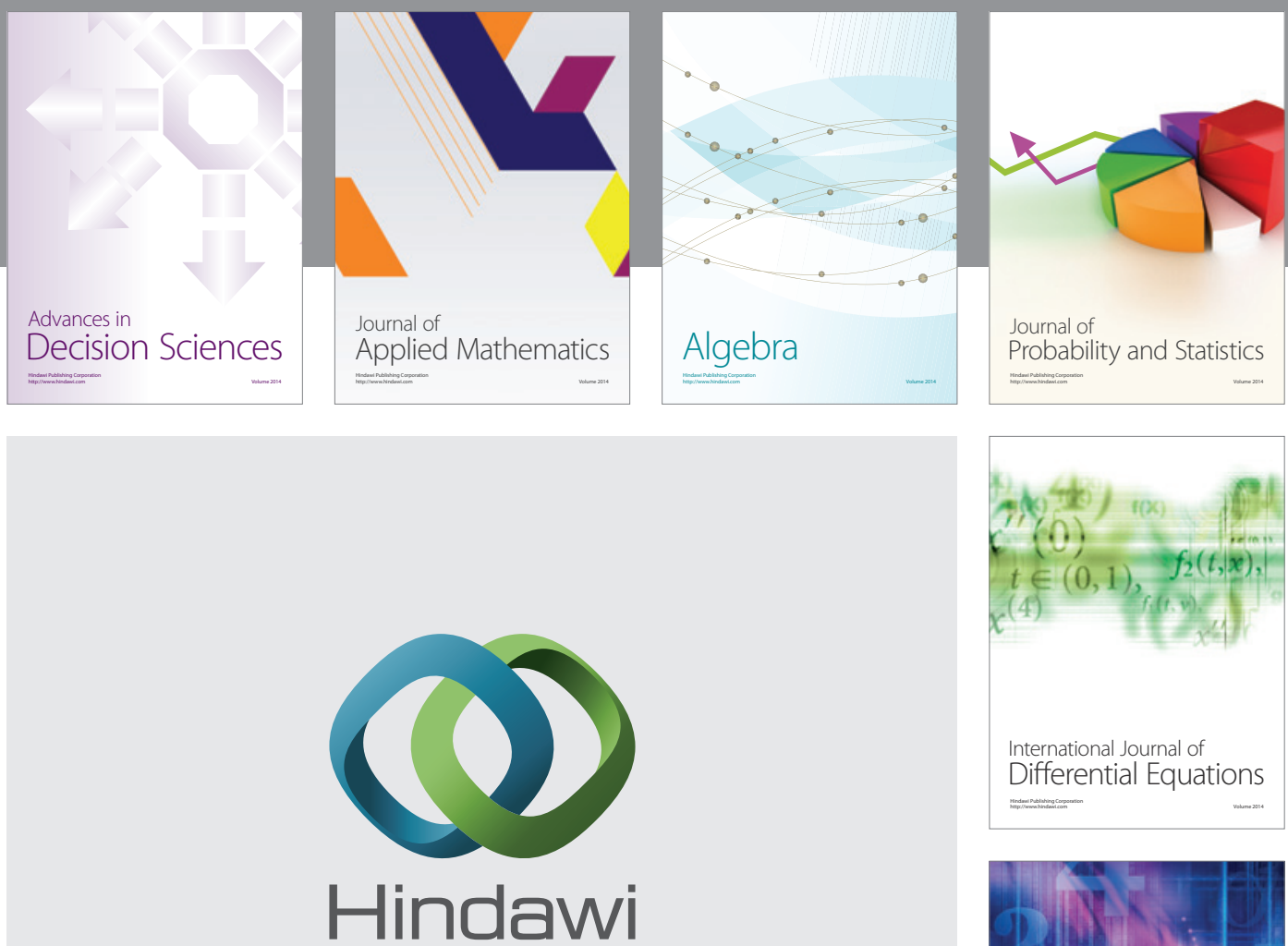

Submit your manuscripts at http://www.hindawi.com
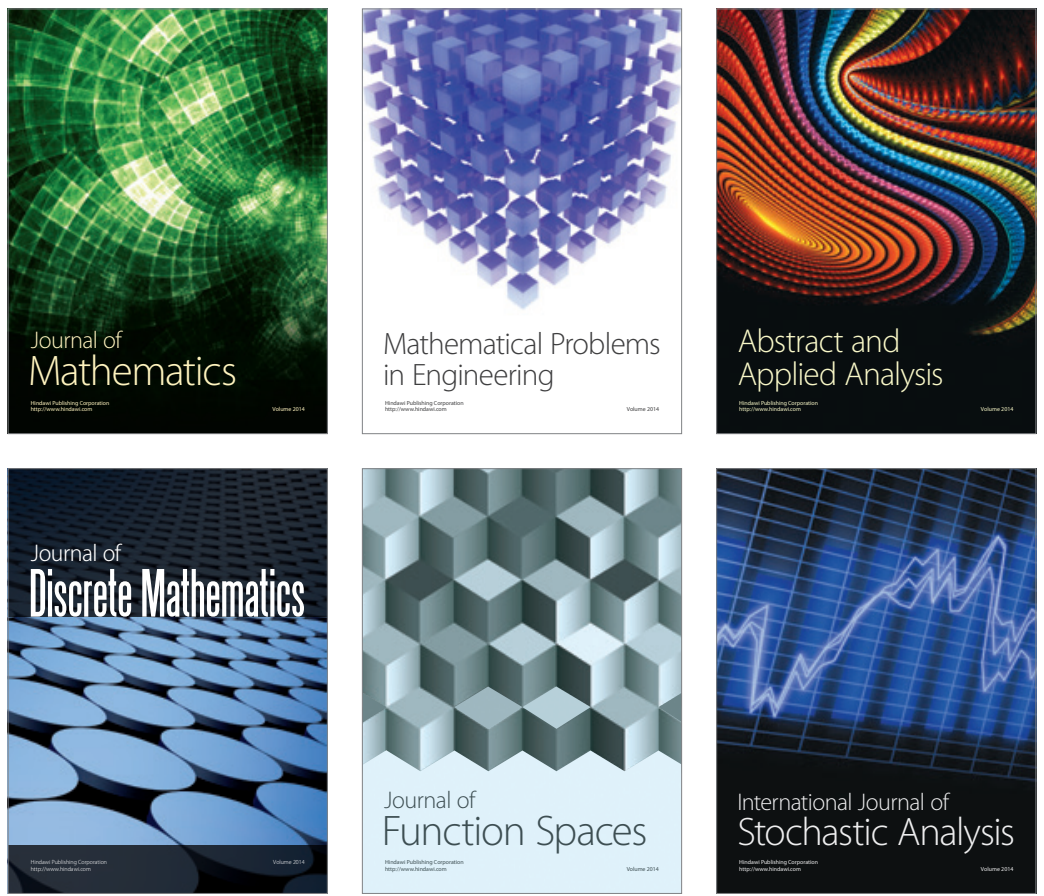

Journal of

Function Spaces

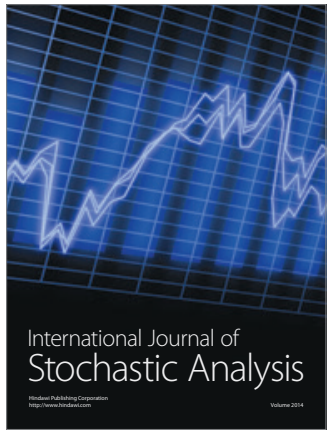

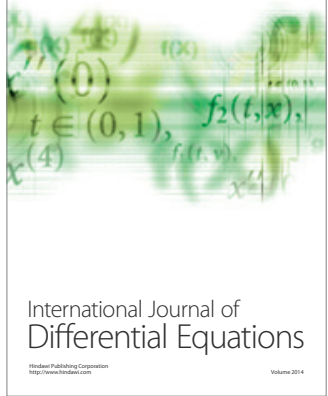
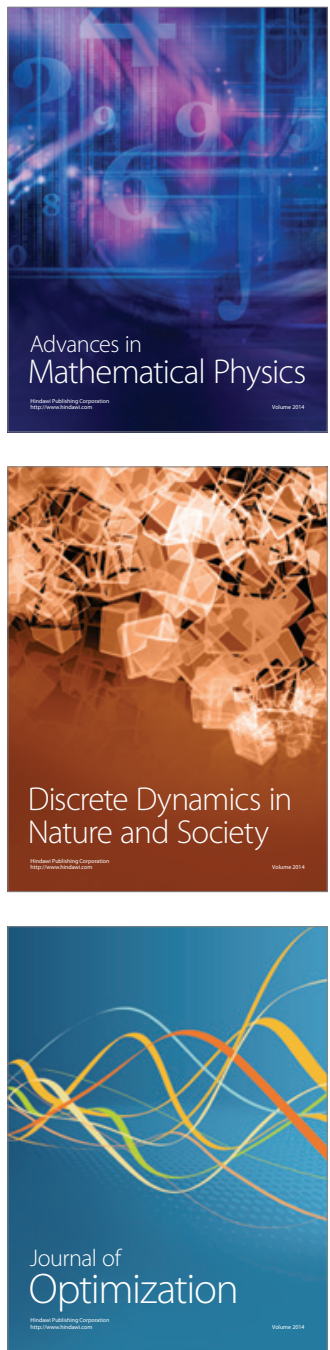